\title{
Context effects in word identification and episodic recognition: A single dissociation
}

\author{
VERONICA STUMPFEL and KIM KIRSNER \\ University of Western Australia, Perth, Australia
}

\begin{abstract}
Two experiments were conducted to evaluate the role of context in word identification and episodic recognition. Each experiment involved a study phase and a test phase. During the study phase, subjects were presented with a mixture of scrambled, semantically anomalous, and semantically integrated sentences. During the test phase, subjects were presented with individual printed words and were required to answer either an episodic-recognition test (Experiment 1) or a wordidentification test (Experiment 2). The results of the word-identification experiment showed that repetition printing, although present under each condition, was insensitive to the contextual manipulation. Performance under episodic-recognition conditions, however, was sensitive to contextual variation; the actual scores ranged from $43 \%$ correct for words presented in scrambled sentences to $60 \%$ correct for words presented in semantically integrated sentences. The results constitute a single dissociation, and they are consistent with the proposition that performance in episodic recognition is sensitive to a form of information that (1) does not influence word identification, and (2) is influenced by variation in contextual factors during encoding.
\end{abstract}

An important and contentious issue in recent research concerns the extent to which performance in wordidentification and episodic-recognition tasks can be explained by reference to a common memory system. In operational terms, the tasks are quite distinct. Subjects in word-identification tasks are typically presented with a degraded stimulus and instructed to name it. They are not required to judge whether or not the stimulus is old or new with reference to an earlier study session, and memory is therefore tested implicitly rather than explicitly. Subjects in episodic-recognition tasks, by contrast, are given an explicit memory test in which they are instructed to indicate whether or not each test word was present in an earlier study session.

The issue is important because evidence that performance in each task is mediated by a common system provides a formal link between perception and memory. According to Jacoby and Brooks (1984), for example, there is no clear distinction between the processes involved in perceptual identification and episodic recognition. They claimed that performance in each task depends on access to information about earlier occurrences of the same stimulus or similar stimuli. There is provision in their account for task-specific effects-where this involves dependence on different types of information-but their basic premise is clear. Performance in each case follows access to memory about specific instances or episodes. The alternative account, that word identification and episodic recognition involve different systems, is widely reflected in the literature, if only in the sense that a wide range

The authors' mailing address is: Department of Psychology, University of Western Australia, Nedlands, Perth, Australia 6009. of word-recognition accounts make no reference to instance-dependent processes in their models (e.g., Forster, 1976; Morton, 1969, 1979).

The specific aim of the experiments reported here was to evaluate the proposition that sentential context selectively influences episodic recognition. The rationale underlying the experiments closely follows that developed by Tulving (1983, chap. 6). Let us suppose that word identification is best thought of as a skill, in which unique sets of word- or morpheme-specific procedures are invoked during word recognition. Suppose, furthermore, that repetition priming reflects some modification of the way in which the system executes the procedures required to identify a particular word; that is, it modifies the "how" of the identification process. This characterization provides a basis for the prediction that prior exposure to a word yields repetition priming. But it makes no provision for interitem effects, and the magnitude of repetition priming should therefore be insensitive to variations in sentential context.

When consideration is given to explicit tests of memory, however, it is plausible to suppose that sentential context is an important variable. If we follow Mandler's (1980) logic, for example, it may be assumed that variations in sentential context influence elaborative structures and, through these, the retrievability of the to-be-remembered item. In practice, however, although there is convincing evidence that sentential manipulations influence both recall (Craik \& Tulving, 1975) and episodic recognition (Fisher \& Craik, 1980), the results suggest that the second of these conclusions is only justified when sentential context is reinstated (Fisher \& Craik, 1980). That is, sentential manipulations do not influence episodic recognition when the test words are tested individually, precisely the con- 
ditions under which testing must be conducted if a meaningful comparison is to be made with word identification.

In summary, the experimental rationale follows the logic of single dissociation. Two tasks are considered, and the underlying hypothesis is that only one of these tasks, episodic recognition, is sensitive to variations in sentential context, the implication being that at least one process or representation is invoked or used exclusively by that task. We have, perforce, restricted testing to individual words, and doubts about the importance of sentential context under these conditions have been addressed by using incidental learning conditions and sampling a broader range of sentential treatments.

Each experiment consisted of a study phase and a test phase. During the study phase, subjects were presented with printed sentences and instructed to rate each sentence for "comprehensibility." Three types of sentence structure were included: scrambled sentences, semantically anomalous sentences, and semantically integrated sentences. The sentence-rating task provided data which could be used to check the sentential manipulation, and an appropriate study task for incidental learning. During the test phase, subjects were required either to indicate whether or not each of a series of individually presented words was old or new with reference to the study list (Experiment 1), or to identify (i.e., name) individual words presented under degraded viewing conditions (Experiment 2).

\section{METHOD}

\section{Design}

The overall design involved manipulation of three variables: (1) test task, involving the contrast between episodic recognition and word identification; (2) repetition status, involving the contrast between old and new words in the test phase; and (3) sentence structure, involving the contrast among three types of sentential organization during the study phase of the experiment. Sixteen subjects were tested under each level of the first variable. Repeated measures were used for the second and the third variables, and the third variable, sentence structure, was nested under the old level of repetition status.

\section{Stimuli}

One hundred semantically related noun-verb pairs (e.g., ZEBRAGRAZE) were selected from KuCera and Francis's (1967) word frequency norms. The words were all low frequency (1-16 per million, inclusive) and five letters in length.

The overall set of 100 noun-verb pairs was divided into four frequencybalanced subsets of 25 word pairs each. Three sentence structures were prepared within each subset such that each word was used once in each structure. The following structures were used: (1) Semantically integrated sentences, which were defined by a subject-verb-object structure in which the test nouns and verbs occupied the first and second positions, respectively (see Example 1). (2) Semantically anomalous sentences, which were constructed from all of the words used in the semantically integrated sentences to produce a mismatch of the relations between the subjectverb-object structures such that meaning was considered to be unlikely (see Example 2). (3) Scrambled sentences, which were constructed from a random allocation of the words composing the original sentence structures, with the constraint that one key noun and one key verb appeared in each sentence (see Example 3).
Example 1: The ZEBRA began to GRAZE on the reserve. Example 2: The SIREN began to BOAST about the casserole dish. Example 3: DWARF new something the same THUMP began invitation.

All of the sentences were eight or nine words in length; the test phase of the experiment therefore included approximately $24 \%$ of the words presented in the sentences, but a much higher proportion of the content words. The test words retained their infinitive forms.

Four sets of 75 sentences each were prepared for the study phase, with sentence structure and item subset being counterbalanced so that each word was included as an item once under each of the four available treatments: in a semantically integrated sentence, in a semantically anomalous sentence, in a scrambled sentence, and in the new condition (where the items were not included at all in the study phase). The noun-verb pairings were preserved for the semantically integrated structure, as shown above, but in the other cases, the words in each pair were included in different sentences, although they remained in the same subset of items.

In the test phase of the episodic-recognition experiment, 250 key words were presented: 50 ( 25 sentences $\times 2$ words per sentence) from each of the four study-phase conditions, plus an additional 50 new words to raise the proportion for that type of item to .40 . The filler items were selected according to the same priniciples with regard to frequency and syntactic category. Following parameter estimation, only 200 words were presented in the test phase of the word-identification experiment, that is, 50 words from each of the four study-phase conditions.

\section{Procedure}

Each subject was tested individually in a sound-attenuated cubicle. The subjects were seated approximately $45 \mathrm{~cm}$ in front of a Tektronix 620 cathode ray oscilloscope. A response box with buttons marked from 1 to 7 was used during the study phase. But during the test phase, subjects used either buttons marked "OLD" or "NEW" (in the episodicrecognition experiment) or named each test word as it was presented (in the word-identification experiment). An intercom system was used for communication between the subject and the experimenter.

The sentences were presented on the oscilloscope, and subjects were required to rate each sentence for comprehensibility on a scale from 1 (low comprehensibility) to 7 (high comprehensibility). Each sentence was presented.for $25 \mathrm{sec}$ or until the subject responded, whichever was shorter. Each subject was informed that there were no right or wrong answers, and that they should therefore respond in their own time. The words were printed in uppercase letters.

The test words in the second phase of the experiment were presented in uppercase letters on the oscilloscope. For episodic recognition, each word was displayed for $10 \mathrm{sec}$ or until the subject responded, whichever was shorter. Subjects were instructed to respond by pressing the OLD button (if the word had been presented in the study phase) or the NEW button (if the word had not been presented in the study phase), and speed and accuracy were given equal emphasis in the instructions.

For word identification, each word was presented for a brief duration and followed immediately by a mask made up of letter fragments. Stimulus onset asynchrony (SOA), the interval between word and mask onset, was determined separately for each subject according to a sequential testing procedure prior to the test phase of the experiment. The mask was presented for $50 \mathrm{msec}$. A new but qualitatively equivalent set of 50 words was used for threshold calculation, and the experimental stimuli were then presented at the selected threshold value. There were only 200 words in the test phase of the word-identification experiment. The word-identification instructions emphasized accuracy, and subjects were encouraged to guess if in doubt.

\section{Subjects}

Thirty-two undergraduate students between the ages of 18 and 21 years participated in each experiment. Four subjects were randomly assigned to each of the eight experimental conditions given by the factorial combination of test task (episodic recognition or word identification) and study list. 
Table 1

Mean Accuracy (Percent Correct) and Reaction Time (in Seconds) as a Function of Experiment, Repetition Status (Old or New), and Sentence Structure

\begin{tabular}{lcccc}
\hline & \multicolumn{3}{c}{ Repetition Status and Sentence Structure } \\
\cline { 2 - 5 } & & \multicolumn{4}{c}{ Old } \\
\cline { 3 - 5 } & & Semantically & $\begin{array}{c}\text { Semantically } \\
\text { Integrated }\end{array}$ \\
\hline $\begin{array}{l}\text { Experiment } \\
\text { Recognition }\end{array}$ & $67.5(2.08)$ & $42.8(3.30)$ & $53.6(2.42)$ & $60.0(1.84)$ \\
$\begin{array}{l}\text { Word } \\
\text { Identification }\end{array}$ & 56.3 & 65.6 & 66.5 & 66.6 \\
\hline
\end{tabular}

\section{RESULTS}

The mean comprehensibility ratings for the scrambled, semantically anomalous, and semantically integrated sentence conditions were $1.77,3.13$, and 6.50 , respectively, indicating that the structural manipulation had the expected effect on comprehensibility. The means for the two experimentally defined groups were virtually identical, and the standard deviations of the means ranged from 0.85 to 1.84 .

\section{Episodic Recognition}

The accuracy and reaction time data for the episodicrecognition group are shown in Table 1 . The reaction time data are based on correct responses only. As expected, sentence structure has a clear effect on both speed and accuracy, and the form of that effect is consistent with the proposition that performance improves as a function of increasing comprehensibility. Even though the test words were tested individually, speed and accuracy were sensitive to the structure of the sentence in which they were embedded during the study phase of the experiment. A one-way ANOVA demonstrated that there were significant differences among the means for the three old conditions for both accuracy $[F(2,30)=19.8, p<.001]$ and reaction time $[F(2,30)=8.12, p<.05]$.

\section{Word Identification}

The pattern of results observed for the wordidentification group is quite different (see Table 1). Repetition priming is present, but the magnitude of the effect is insensitive to sentence structure. A one-way ANOVA indicated that there are significant differences among the complete set of new and old means $[F(3,45)=7.8$, $p<.05$ and .001$]$, but as the confidence interval $(p=$ .05 ) for these data is 3.04 , the hypothesis that there are no reliable differences among the old conditions may be accepted.

\section{DISCUSSION}

\section{Sentence Effects in Episodic Recognition}

Although the episodic-recognition results are not consistent with previous research involving sentential manipulation, this is not entirely unexpected, given the number of procedural differences between the studies concerned. The most obvious differences between the present study and that conducted by Fisher and Craik (1980) were as follows. First, the sentential manipulation used by Fisher and Craik involved a comparison between simple and complex sentences, where the complex sentences included additional word or phrase modifiers. In the present case, however, the manipulation was more dramatic, involving comparisons between sentences in which meaning and syntax were intact, sentences where syntax and meaning were intact and degraded, respectively, and sentences where both syntax and meaning were degraded. Second, whereas the subjects in Fisher and Craik's study were instructed to remember the study material, our subjects were given an incidental orienting task. Third, whereas the study material was presented auditorily in Fisher and Craik's (1980) study, our study material was presented visually. The second and third of these explanations can probably be discounted, however. The fact that Fisher and Craik (1980) found a sentential effect when they tested subjects under sentence conditions demonstrates that their subjects had encoded information about sentence structure during the study phase, and we have subsequently replicated the results reported here using auditory and visual presentation during the study and test phases, respectively (Stumpfel, 1983).

\section{Word Identification and Episodic Recognition: \\ A Single Dissociation}

The results provide a straightforward illustration of single dissociation. Whereas sentential context influenced performance in episodic recognition, no differential effect was observed for word identification. The outcome is consistent with an account in which episodic recognition, at least, involves one unique process. That process appears to involve access to a form of information where strength or accessibility is sensitive to contextual manipulation during the study phase. Performance in word identification does not appear to rely on or use this information, and the outcome therefore constitutes a single dissociation.

The relationship between the results reported here and those reported by Jacoby (1983, Experiment 1) requires further comment. Superficially, they both involve context, yet they demonstrate dissociation and association between word identification and episodic recognition, respectively. However, the sense in which context is being used is quite different in the two cases. In Jacoby's study, the relevant manipulation involved the proportion of old items presented during the study phase (10\% vs. $90 \%$ ), and subjects in the latter treatment were informed prior to the test phase that it would consist of words that they had just read (whereas subjects in the $10 \%$ group were informed that the test would consist of new words). Thus, the critical context in Jacoby's case consists of the entire list or, possibly, the session; whereas in our case, it is restricted to the structure of the sentence in which the word was presented during the study phase. Moreover, the fact that Jacoby's manipulation influenced performance from the first trial (Jacoby \& Witherspoon, 1982) suggests that it reflects a strategic change in which subjects invoke, for example, postlexical processes.

Finally, it may be noted that the cooccurrence of association and dissociation between two tasks is only paradoxical if it is assumed that there is a one-to-one mapping between the task and process levels. If it is assumed, for example, that word identification and episodic recognition each involve access to a number of processes, it follows that association represents intersection in the process domain (i.e., cases where one process influences both tasks) and dissociation represents nonintersection in the processing domain (i.e., cases where one process is unique to one of the tasks). Thus, the fact that the proportion of old items (or instructions about the proportion of old items) influences episodic recognition and word identification (Jacoby, 1983) whereas sentential context selectively influences episodic recognition is consistent with the following summary. First, Process A, which is sensitive to the proportion of old items (or instructions about the proportion of old items), operates in word identification and episodic recognition. Second, Process B, which is sensitive to sentential context, operates selectively in episodic recognition. The results are not incompatible.

\section{Single Dissociation and Differential Sensitivity}

Although single dissociation is sometimes criticized on the ground that it may simply reflect differential sensitivity between tasks, that criticism is difficult to sustain in the present case. Where modality is concerned, for example, it is apparent that word recognition is more sensi- 
tive to changes in stimulus class than is episodic recognition. Kirsner, Milech, and Standen (1983), for example, found that repetition priming was reduced by approximately $50 \%$ when there was a modality change between the study and test phases, and more severe effects have been observed in other studies (e.g., Jacoby \& Dallas, 1981; Morton, 1979). In episodic recognition, however, the cost of changing modality is either negligible (Kirsner, 1974) or absent altogether (Siple, Fischer, \& Bellugi, 1977).

In summary, a single dissociation was observed, and the form of that dissociation was consistent with the proposition that a process which operates under sentential conditions produces consequences which selectively influence performance in episodic recognition.

\section{REFERENCES}

Craik, F. I. M., \& Tulving, E. (1975). Depth of processing and the retention of words in episodic memory. Journal of Experimental Psychology: General, 104, 269-294.

Fisher, R. P., \& CraIK, F. I. M. (1980). The effects of elaboration on recognition memory. Memory \& Cognition, 8, 400-404.

Forster, K. I. (1976). Accessing the mental lexicon. In R. J. Wales \& E. Walker (Eds.), New approaches to language mechanisms. Amsterdam: North Holland.

JACOBY, L. L. (1983). Perceptual enhancement: Persistent effects of an experience. Journal of Experimental Psychology: Learning, Memory, and Cognition, 9, 21-38.

JACOBY, L. L., \& BROOKS, L. R. (1984). Nonanalytic cognition: Memory, perception and concept learning. In G. H. Bower (Ed.), The psychology of learning and motivation: Advances in research and theory (Vol. 18). New York: Academic Press.

JACOBY, L. L., \& DALlAS, M. (1981). On the relationship between auto- biographical memory and perceptual learning. Journal of Experimental Psychology, 110, 306-340.

JACOBY, L. L., \& WITHERSPOON, D. (1982). Remembering without awareness. Canadian Journal of Psychology, 36, 300-324.

KIRSNER, K. (1974). Modality differences in recognition memory for words and their attributes. Journal of Experimental Psychology, 102, 579-584.

Kirsner, K., Milech, D., \& Standen, P. (1983). Common and modality-specific units in the mental lexicon. Memory \& Cognition, 11, 621-630.

KuČERA, H., \& Francis, W. N. (1967). Computational analysis of present-day American English. Providence, RI: Brown University Press.

Mandler, G. (1980). Recognizing: The judgment of occurrence. Psychological Review, 87, 252-271.

Morton, J. (1969). Interaction of information in word recognition. Psychological Review, 76, 165-178.

MoRTON, J. (1979). Facilitation in word recognition: Experiments causing change in the logogen model. In P. A. Kolers, M. E. Wrolstad, \& H. Bouma (Eds.), Processing of visible language. New York: Plenum Press.

SiPle, P., Fischer, S. D., \& Bellugi, U. (1977). Memory for nonsemantic attributes of American sign language signs and English words. Journal of Verbal Learning \& Verbal Behavior, 16, 561-574.

STUMPFEL, V. (1983). Modality and sentence comprehensibility effects in perceptual identificaton and memory. Unpublished honors thesis, University of Western Australia, Perth.

Tulving, E. (1983). Elements of episodic memory. New York: Oxford University Press.

(Manuscript received for publication December 5, 1985.) 